Proceedings of SALT 22: 584-603, 2012

\title{
Degrees of countability: A mereotopological approach to the mass/count distinction*
}

\author{
Scott Grimm \\ Universitat Pompeu Fabra
}

\begin{abstract}
Most formal semantic treatments of countability aim to account for a binary count/non-count distinction through the use of mereology, or part-structures. This paper discusses data from Welsh, which possesses three categories of grammatical number, distinguishing a collective/singulative class under which fall entity types such as COLLECTIVE AGGREGATES (swarming insects, vegetation) and GRANULAR AGGREGATES (sand). I show that standard mereological accounts turn out not to be sufficiently expressive to account for this broader typological data. I then argue that it is necessary to enrich mereology with connection relations that model ways in which the referents of nouns may come together, resulting in the more expressive MEREOTOPOLOGY. I show that this extension leads to faithfully modeling the degrees of countability found in Welsh and overcomes well-known problems for classical mereological accounts, e.g., the "minimal parts" problem.
\end{abstract}

Keywords: mass/count, mereology, topology, collective, singulative, Welsh

\section{Introduction}

The traditional semantic analysis of the mass/count distinction, stemming from Quine (1960) and Link (1983), models a binary countable/non-countable contrast within a mereological framework (or other equivalent part-structures) via several semantic properties, such as ATOMICITY, CUMULATIVITY and DIVISIVENESS. This approach has been rightly influential: it is able to model a variety of different types of entities (singular, plural, mass) which is elusive in standard set theory, and is further able to derive the grammatical behavior of nouns as either countable or non-countable through whether the nominal predicate satisfies different properties, e.g., atomicity for countable nouns or divisiveness for non-countable nouns. Despite these gains, using classical extensional mereology to model countability phenomena

* This work stems from chapter 4 of my dissertation (Grimm 2012) and I would like to thank my committee Beth Levin, Donka Farkas, Paul Kiparsky and Chris Potts for their input throughout that period. Many thanks also to Jenny Doetjes, Hana Filip, Manfred Krifka, Dan Lassiter, Laia Mayol, Louise McNally, Henriëtte de Swart and Henk Zeevat for valuable feedback at different points during this project and to the audience at SALT 22.

(C)2012 Grimm 
A mereotopological approach to the mass/count distinction

is beset by several persistent problems. For instance, it has been widely noted that the semantic properties made available by mereology fail to fully predict which nouns are countable and which are non-countable (see Taylor 1977; Zucchi \& White 2001, among others).

This paper retains the spirit of the traditional semantic approach grounding countability distinctions in semantic distinctions, yet argues that (i) countability is a richer phenonmenon than a binary countable/non-countable contrast, and, in part because of this but also because of theory-internal difficulties with mereology, that (ii) a richer variant of the mereological framework extended with topological relations is necessary. First, in section 2, I argue that modeling a binary countable/non-countable distinction is not sufficient as an emprical target. Although English presents a binary contrast, other languages may make a richer set of countability distinctions, as is shown for Welsh, and thus there are actually degrees of countability that must be modeled. In section 3, I argue that the failure of mereology to adequately model countability distinctions stems from foundational inadequacies of mereology, which have attracted much discussion in the philosophy and ontological modeling literatures. Standard mereology is not capable of modeling what it means to be a whole individual, nor capture relations between individuals, both of which are necessary for modeling countability. Section 3 proposes a topological extension of the mereological framework that is able to properly model different entity types such as substances, whole individuals, and varieties of collectives. In section 4, I apply this system to the grammatical number systems of English and Welsh.

\section{Countability and the collective-unit distinction in Welsh}

Welsh, like English, displays a contrast between countable and non-countable nouns. Countable nouns show a regular alternation between singular and plural values while non-countable nouns are "not usually found in the plural" (King 2003: 35). Examples of countable and non-countable nouns are given in (1a) and (1b), respectively. This contrast is also supported by co-occurrence behavior with quantifiers such as amryw 'several', which co-occurs with countable plural nouns, but not with non-countable nouns, as shown in (2) (Jones \& Thomas 1977: 176).

(1) a. Countable (plural-coded) nouns: afal/afalau 'apple/apples'; cadairl cadairiau 'chair/chairs'; dyn/dynion 'man/men'; merch/merched 'girl/girls'

b. Non-countable nouns: glo 'coal'; 'menyn 'butter'; llefrith 'milk'

(2) amryw o 'r llyfrau/*eira

'several of the books/*snow'

Yet, Welsh, unlike English, possesses two distinct types of countable nouns. In addition to nouns where the plural value is morphologically coded, as in (1a), 


\begin{tabular}{|cccc|}
\hline Countability Category & Singular & Plural & Gloss \\
\hline \hline Singular/Plural & cadair & cadairiau & 'chair' \\
Collective/Unit & cacyn-en & cacwn & 'hornet' \\
\hline \hline Non-Count & \multicolumn{2}{c|}{ llefrith } & 'milk' \\
\hline
\end{tabular}

Table 1 The Tripartite Grammatical Number System of Welsh

there is a second type of noun where the singular value is morphologically coded. I will follow the terminology of King (2003) and designate the latter type as the COLLECTIVE/UNIT class and call the morpheme which codes the singular value the SINGULATIVE. Typical examples of nouns in this class are given in (3). A notional distinction accompanies this formal distinction: nouns in the collective/unit class are entities which habitually co-occur in the world. Stolz (2001) provides a detailed analysis of the lexical items which comprise the collective/unit class in Welsh, noting that they are restricted to a small number of entity types, namely what I call COLLECTIVE AGGREGATES, such as small animals, insects, vegetation or cereals, and what I call GRANULAR AGGREGATES, such as sand. Stolz concludes that nouns in this class designate entities which are "saliently perceived as collectivities rather than "individuals", (2001: 65). Table 1 summarizes the three different categories in Welsh's number system: singular/plural, collective/unit, and non-countable.

(3) Collective/Unit nouns: cacwn/cacyn-en 'hornets/hornet'; picwn/picwn-en 'bees/bee', chwain/chwann-en 'flea/fleas'; dincod/dincod-yn 'seeds/a seed'; ceirch/ceirch-en 'oats/an oat'; chwynn/chwynn-yn 'weeds/weed'; cnau/cneuen 'nuts/nut'; tywod/tywod-yn 'sand/grain of sand'; marwor/marwor-yn 'embers/an ember'; llwch/llych-yn 'dust/speck of dust'

The first question to be addressed is whether this additional category of collectives is actually relevant for understanding the distinction between countable and non-countable nouns. Coming from the perspective of a binary count/non-count contrast, one could question whether these three categories could not be wedged into a binary mold. Two arguments indicate that such a reduction is unwanted.

First, the entity types in the collective/unit class are notionally distinct from those typically associated with countable and non-countable nouns. The core classes of entity types realized as non-countable nouns and countable nouns in Welsh and English are essentially identical, namely liquids/substances and individuated objects, respectively. Yet, the collective class does not fit comfortably in either. Many members of the collective class would make for extremely dubious individuals, for instance in the case of the cognates of dust or sand, a point reinforced by the fact 
A mereotopological approach to the mass/count distinction

that these nouns are non-countable in English.

A more serious objection to conflating the collective class with simple plural nouns is that it is common for collective nouns to make a three-way distinction between collective, singular and plural values, which would be unexpected if the collective and plural values coincided. An example from Welsh is given in (4) (from Stolz 2001: 70) where the collective and plural forms clearly designate distinct meanings: the pluralized singulative form designates a set of individual pieces in contrast to, e.g., a heap of grain.

$$
\begin{aligned}
& \text { grawn / gron-yn / gron-ynn-au } \\
& \text { grain / grain-SING / grain-SG-PL } \\
& \text { 'grain / a single grain / grains' }
\end{aligned}
$$

Another possibility is that collectives could be treated as related to "group" nouns (in the sense of Landman 1989); however, there are two arguments against collapsing the collective class with group nouns. Again, the collective class differs notionally from characteristic group nouns, such as committee, which designate social bodies or organizations. Further, group nouns in Welsh are a grammatically distinct class of nouns: the singular value is morphologically uncoded while the plural value is moprhologically coded, similar to standard countable nouns, but the singular noun form requires plural agreement with the verb.

The implication for theories of countability, then, is that a binary categorization of nouns into countable and non-countable may be necessary, but is not sufficient. Both a simple grammatical distinction between nouns compatible with number coding and those not, as well as a notional distinction between individuals and non-individuals, oversimplifies the typological space. Semantic theories of countability must also account for collective entities, modeling how they habitually come together.

\section{A mereotopological treatment of countability}

A central advantage of using mereology for linguistic applications is its liberal view of what an individual designates. A mereological "individual" may correspond to any number of entities in the world which are disjoint and even widely spatially separated. Nor does an individual necessarily have any boundaries-from the viewpoint of mereology, "an individual is simply a segment of the world of experience, and its boundaries may be complex to any degree" (Goodman 1951: 42). Thus, an individual can be any section of the world, or combinations thereof. This flexibility as to what qualifies as an individual is the source of its usefulness for the treatment of countability, since it is able to model plural and substance "individuals". Yet, while mereology may represent plural and substance entities where set theory cannot, much recent work in philosophy and ontological modeling (Smith 1996, Casati \& 
Varzi 1999) has pointed out significant gaps between intuitions about how objects stand in the world and what mereology delivers: the concept of a whole object or relations holding between individuals are inexpressible in classical mereology. These works propose to remedy the situation by extending standard mereology with connectedness relations, constructing systems of "mereotopology".

This section lays out the classic mereological treatment of countability and discusses its shortcomings. I will argue that many of these shortcomings are related to the general shortcomings of mereology. I then pursue a topological extension of mereology that will account for (i) the core data of objects and substances which has proved problematic to model over the last decades and (ii) the richer data concerning collectives elaborated in section 2 .

Before proceeding, it is worth warding off terminological confusion concerning the use of individual. Its use in the mereological literature is clearly different from the pre-theoretic sense, where it refers to well-defined physical objects in opposition to stuff. To eliminate any confusion, I will refer to individuals in the mereological sense as M-INDIVIDUALS and retain INDIVIDUAL for the pre-theoretic use.

\subsection{Mereology and countability: The classic account and its shortcomings}

I first lay out a standard axiomatization of mereology which has been assumed in canonical works on countability in the formal semantic tradition, such as Link 1983, Krifka 1989, and Landman 1989, before proceeding to enrich it. The lexical core of a mereological theory is provided by a treatment of the "part-of" relationship, $\leq$. There is wide consensus that to conform to its intuitive use, the part relation must be reflexive, antisymmetric and transitive, given as the first three axioms. ${ }^{1}$ The relations OVERLAP and PROPER PART can then be defined as in D 1-D 2.

M $1 x \leq x$ (Reflexivity)

M $2 x \leq y \wedge y \leq x \rightarrow x=y$ (Antisymmetry)

M $3 x \leq y \wedge y \leq z \rightarrow x \leq z$ (Transitivity)

D $1 O(x, y)=\operatorname{def} \exists z(z \leq x \wedge z \leq y)$ (Overlap)

D $2 x<y={ }_{\text {def }} x \leq y \wedge \neg(y \leq x)$ (Proper Part)

The basic axioms of mereology are extended in two ways. The first type of extension restricts the manner in which an m-individual can be decomposed into different parts through the SUPPLEMENTATION axiom (also known as the REMAINDER PRINCIPLE). This requires that if an object $a$ has a proper part $b$, then there is also another proper part, $c$, which doesn't overlap with $b$. This excludes in particular

1 All the axioms given here are universally quantified. 
models where an m-individual is decomposed into a single proper part. The second type of extension concerns adding individuals together. The sum operation is defined in D 3 and notation for a binary sum operator and a generalized sum operator is given in D 4 and D 5, respectively. The traditional principle of mereology, again not uncontroversial as will be discussed further in section 3.2, is that for any two individuals, there is also a sum individual comprised of those two individuals. Another way to state this is that the part structures that are of interest are those which are closed under sum formation, which is ensured by requiring every set of m-individuals to have a unique sum, for which the axiom is given in M 5.

M $4 x<y \rightarrow \exists z(z \leq y \wedge \neg O(z, x))$ (Supplementation)

D $3 \operatorname{sum}(x, X)={ }_{\operatorname{def}} \forall y[X(y) \rightarrow y \leq x] \wedge \forall z\left[z \leq x \rightarrow \exists z^{\prime}\left[X\left(z^{\prime}\right) \wedge O\left(z, z^{\prime}\right)\right]\right]$

(A sum of a set $X$ is a thing that contains everything in $X$ and whose parts each overlap with something in $X$.)

D $4 x \oplus y=\operatorname{def} \boldsymbol{\imath} z \operatorname{sum}(z,\{x, y\})$

D $5 \oplus X={ }_{\text {def }} \boldsymbol{l} z \operatorname{sum}(z, X)$ where $X$ is any set

M $5 \forall X[X \neq \emptyset \rightarrow \exists ! z \operatorname{sum}(z, X)]$ (Uniqueness of Sums)

The theory arrived at through these different axioms is the standard version of mereology used in philosophy and semantic theory, known in the literature as General Extensional Mereology. This system is expressive enough to represent the standard Boolean operators, such as DIFFERENCE and COMPLEMENT, given in D 6 and D 7, respectively.

D $6 x-y={ }_{\operatorname{def}} \boldsymbol{\imath} z \forall w(w \leq z \leftrightarrow(w \leq x \wedge \neg O(w, y)))$ (Difference)

D $7-x={ }_{\text {eff }} \imath z \forall w(w \leq z \leftrightarrow \neg O(w, x))$ (Complement)

In addition to being tolerant of different types of entities (plurals, substances), mereology provides a natural framework in which to elegantly model properties of entities through their behavior in terms of the part relation. Properties such as CUMULATIVITY, DIVISIVENESS, or ATOMICITY have been argued to characterize the countability status of different predicates. The relation between these properties and the classification of nouns as countable or non-countable is controversial for each property. For instance, since Quine (1960), cumulativity and divisiveness, given in D 8 and D 9, have been typically considered as relevant for non-countable nouns. Yet, cumulativity characterizes not only non-countable predicates such as water, but also plural predicates, such as cows. Consequently, cumulativity does not distinguish countable from non-countable nouns and so it is unclear if this property by itself has much to do with countability per se.

D 8 Cumulative $(P)=\forall x \forall y[P(x) \wedge P(y) \rightarrow P(x \oplus y)]$ 
D 9 Divisive $(P)=\forall x[P(x) \rightarrow \forall y[y<x \rightarrow P(y)]]$

It is initially plausible that divisiveness characterizes non-countability. For instance, assuming that mud is a divisive predicate, for a given quantity of mud, it follows that every part of this mud is again mud, whereby it is clear why such a predicate is non-countable: it does not provide a stable unit for counting. An entity that could be described as one mud could by the same rights be described as any arbitrary number of muds, since all its parts are mud as well. Yet, as is well-known, grounding non-countability in the property of divisiveness produces many counterexamples when applied systematically across the lexicon. One effective illustration comes from non-countable nouns such as soup (Taylor 1977). If soup is divisive and soup is true of some $a$, then any part of $a$, say $a_{1}$, is also soup. Yet, should a carrot be in the soup, the part of the soup which consists of some interior chunk of the carrot, does not count as an instance of soup.

The third property, ATOMICITY, is often intended to characterize discrete individuals. From a mereological point of view, an atom is an individual which has no proper parts, as given in D 10. Some approaches simply have models that are atomistic (Link 1983; Chierchia 1998a). This is implemented by adding an axiom requiring everything to be ultimately composed of atoms, as in M 6. A different approach defines atoms relative to a property, as given in D 11. Given this definition, one can further define what it means for a predicate to be atomic, as in D 12 from Krifka (1989), where Atomic $_{P}$ indicates atomic relative to $P$.

D $10 \operatorname{Atom}(x) \leftrightarrow \neg \exists y(y<x)($ Atom $)$

M $6 \forall x \exists y(y \leq x \wedge \neg \exists z(z<y))$ (Atomicity)

D 11 Atomic $(x, P)=P(x) \wedge \neg \exists y[y<x \wedge P(y)]$ (Atomic relative to a property)

D $12 \operatorname{Atomic}(P)=\forall x[P(x) \rightarrow \exists y[y \leq x \wedge \operatorname{Atomic}(y, P)]]$ (Atomic predicate)

While most countable nouns can be reasonably treated as atomic predicates, there are still several classes of problematic nouns, discussed at length in Zucchi \& White 2001 and Rothstein 2010. One such class of nouns is exemplified by fence, where a part of a fence may again merit being designated as a fence. Geometrical terms such as line or grouping terms such as sequence show the same behavior. Terms of indefinite reference, such as event, thing, a quantity of $X$ also fail to be atomic.

In summary, the wide adoption of these properties is an indication of their intuitive appeal and it is also clear that these are sensible properties to propose from the mereological perspective, as they all exploit the part relation in a natural way. Yet, decades of research have shown that the correspondence between these properties and countability phenomena is imperfect. 
A mereotopological approach to the mass/count distinction

In addition to the well-known problems just discussed, there are problems with the foundations of mereology itself. These are not discussed in the literature on countability, but the philosophical and ontological modeling literature has argued that mereology's use of mereological individuals simply does not provide a satisfying account of ordinary individual objects, which is of course one of mereology's aims as a model of the world. While mereology is often said to be a theory of the part-whole relation, in truth it is only a theory of parthood and does not give a sufficient account of whole objects. ${ }^{2}$ The sum of two entities $a \oplus b$ is often casually equated with the "whole" to which the parts $a$ and $b$ belong. Yet, sums simply cannot be equated with wholes - the sum of the Empire State Building and the letter ' $z$ ' exists by the same rights as the sum of two halves of an apple making a whole apple. As Casati \& Varzi (1999: 51) state, "it is impossible ... to draw a distinction between 'good' and 'bad' wholes." These criticisms of mereology's expressive power are in turn relevant for an adequate treatment of the countability distinctions of different nominal predicates.

At its most intuitive, the distinction between countable and non-countable nouns is tied to the distinction between objects and substances. This distinction is obviously too coarse for, e.g., non-countable nouns in English, but at least this must be covered. Objects, in turn, are intuitively whole objects, as Soja, Carey \& Spelke (1991) and Prasada, Ferenz \& Haskell (2002) have shown in the developmental and cognitive literatures. If the criticism that mereology doesn't provide a satisfactory treatment of whole objects is well-placed, then it is clear from where (at least some of) the problems that arose in modeling countability stem: mereology is not suited to represent (at least) one side of object/substance divide.

It also becomes clear then why higher order properties such as atomic or quantized fail as characterizations of objects: they are an attempt to characterize wholes in a theory which is unable to carry out that task. Yet, since the part relation does not say anything about what a whole object is, these properties, based on the part relation, do not provide a characterization of what a whole is either. Instead, these properties demonstrate the awkward fit between the explananda and the characterization in terms of parts. Atomicity/quantization leads to finding parts when one doesn't want them, as in the case of fence. On the other hand, divisiveness leads to not finding parts where one needs them, e.g., according to divisiveness, one should (but doesn't) find increasing smaller parts of soup which count as soup.

2 This is one of the criticisms put forth by Moltmann (1997) in relation to the countable/non-countable distinction, an important precedent for the view I argue for. Moltmann also proposes that whole objects must be formally recognized, although her account rejects mereology altogether. See Pianesi 2002 for a critical view on Moltmann's rejection of mereology. 


\subsection{Extending mereology: Part-whole structures and connectedness}

I will now extend mereology with topological relations, following in many respects the discussion of strengthening mereology with topology found in Casati \& Varzi 1999. The fundamental change is adding the relationship of CONNECTEDNESS, which then interacts with the different definitions and axioms of standard mereology. The intuitive definition of being connected is that two entities are connected if they share a common boundary. An alternate characterization is that if two entities are disjoint, then they are SEPARATED. Accordingly, any entities that are not separated are connected. It is easy to illustrate with some examples from the physical world. My chair is touching the floor, and therefore qualifies as connected to the floor. The upper two-thirds of my mug is connected to the lower two-thirds; in fact they overlap, sharing a common part in the middle third. My mug and the Taj Mahal are, however, separated (although they do have a mereological sum).

Some basic requirements are that the relation C, CONNECTED, is reflexive and symmetrical. These are added as axioms in $\mathrm{T} 1$ and $\mathrm{T} 2$. There are some further intuitive interactions with the mereological relations part, $\leq$, and overlap, $\mathrm{O}$, that Casati \& Varzi (1999) note. First, the axiom in T 3 ensures that parthood implies connectedness. From T 3 the relation in (5) follows, whereby overlap implies connectedness.

T $1 C(x, x)$ (Reflexivity)

T $2 C(x, y) \rightarrow C(y, x)$ (Symmetry)

T $3 x \leq y \rightarrow \forall z(C(x, z) \rightarrow C(z, y))$

$$
O(x, y) \rightarrow C(x, y)
$$

This extension of mereology is able to express standard notions of topology, such as interior, closure or boundary, and a wide array of mereotopological properties that make fine-grained distinctions as to different configurations that objects may be in. I show now how it can be exploited to model whole objects and different connectedness relations holding between entites, substances and aggregates.

\subsection{Defining wholes}

Returning to one of the primary motivations for adding topological relations, it is now possible to distinguish entities which form one whole piece as opposed to arbitrary sums. This is achieved through recognizing "self-connected" (SC) entities-individuals which cannot be divided into two separated parts. The basic definition is given in D 13. 
A mereotopological approach to the mass/count distinction

D $13 S C(x)={ }_{\operatorname{def}} \forall y, z(\forall w(O(w, x) \leftrightarrow(O(w, y) \vee O(w, z))) \rightarrow C(y, z))$

(An entity $x$ is self-connected if any two parts that make up the whole of $x$ are connected to each other.)

We can now distinguish between objects which come in one piece as opposed to scattered objects. For instance, suppose our domain of discourse contains four individuals, where $a$ and $b$ are two halves of a sphere and $c$ and $d$ are my left shoe and the Eiffel Tower. Then we can distinguish the sum $x=a \oplus b$ from the sum $y=c \oplus d$. $x$ is a whole, i.e., $\mathrm{SC}(\mathrm{x})$ is true, while the "scattered individual" $y$ is not a whole, and $\mathrm{SC}(\mathrm{y})$ is false.

Yet, this definition does not rule out a sum of entities which just "touch"-a stronger notion is needed to do justice to the notion of a whole. Casati \& Varzi (1999) supply two stronger self-connectedness properties, which rely on the ability of mereotopology to discuss the interior of an object. First, I give the definition of the property of INTERNAL PART (IP) in D 14. The defintion of interior is given in D 15 as the sum of an m-individual's internal parts. Now, a stronger requirement on self-connectedness can be stated wherein an entity's interior must be connected, given in D 16, which correctly rules out entities which just touch on their borders.

D $14 \operatorname{IP}(x, y)={ }_{\operatorname{def}} x \leq y \wedge \forall z(C(z, x) \rightarrow O(z, y))$ (Internal Part)

D $15 i x={ }_{\text {def }} \bigoplus X$ where $X=\{y: I P(y, x)=$ True $\}$ (interior)

\section{D $16 \operatorname{SSC}(x)={ }_{\operatorname{def}} S C(x) \wedge S C($ ix $)($ Strongly Self-Connected $)$}

If an individual has the property of being strongly self-connected, that still does not guarantee that it is also a whole in the intuitive sense. Consider the mindividual, i.e., "the segment of the world of experience" according to Goodman, that corresponds to exactly one half of a sphere. The half sphere qualifies as strongly self-connected, but it does not intuitively qualify as a whole, for that would be the entire sphere. Since we are interested in unity, we can define being maximally connected relative to a property, which provides our final definition of what it means to be a whole. If an entity satisfies Maximally Strongly Self-Connected (MSSC) as given in D 17, then that entity is the largest entity satisfying that property which is self-connected. Although such distinctions may seem subtle, they have very welcome consequences for modeling countability.

D $17 \operatorname{MSSC}(x, P)=\operatorname{def}_{\operatorname{def}}(x) \wedge S S C(x) \wedge \forall y(P(y) \wedge S S C(y) \wedge O(y, x) \leftrightarrow y \leq x)$ (Maximally Strongly Self-Connected relative to a Property)

(An m-individual is Maximally Strongly Self-Connected relative to a property if ( $i$ ) every (interior) part of the individual is connected to (overlaps) the whole (Strongly 
Self-Connected) and (ii) anything else which has the same property and overlaps it is once again part of it (Maximality).)

I can now treat predicates which refer to individuals (in the pre-theoretical, nonmereological sense) as those predicates which are restricted to designating whole objects or sums thereof. Very little must change about the way that we represent the meaning of count nouns, except for the substitution of the property of being a whole object, viz. MSSC, for the property atomicity. For instance, the condition given in (6) of an INDIVIDUAL-predicate is an adaptation of the definition of an atomic predicate in D 12. This condition prevents strongly connected (overlapping) individuals from being in the extension of an individual predicate. The singular property of, for instance, $\operatorname{dog}$, simply picks out individual (whole) dogs.

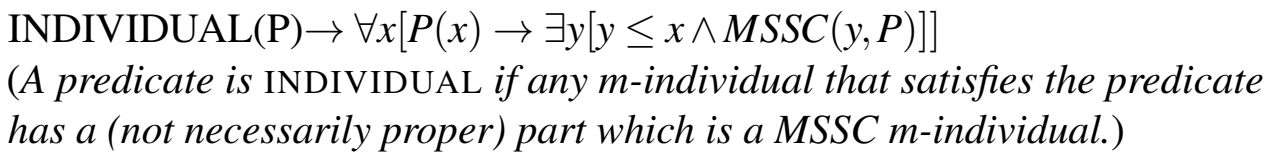

Using the full strength of maximally strongly self-connected (MSSC) m-individuals overcomes the problems for atomicity related to predicates such as fence or twig, discussed in section 3.1. As a result of the maximality condition, the problems with predicates such as fence simply do not arise: INDIVIDUAL-predicates restrict their reference to pick out only MSSC m-individuals, which by definition will be in each case the largest individual satisfying, for instance, fence. A part of a fence may have all the requisite features to satisfy the predicate fence, but it will not be a MSSC-individual. Thus, when a fence is uttered, this will pick out only the whole object, although there are a variety of ways to pick out portions of the fence, which, if considered in isolation, would also qualify as an instance of the kind fence. This explanation extends to other examples problematic for atomicity, such as twig and line or even the more fanciful example of the Pope's crown (Wiggins 1980: 73), which is problematic since it contains three smaller crowns. When using MSSC as the foundational property of countable nouns, these problems simply don't arise.

\subsection{Varieties of connectedness}

The basic connectedness relation $\mathrm{C}$ is taken as primitive, from which different degrees of connectedness can be defined. The two primary types of connectedness turn upon whether the two connected entities overlap in a substantive sense or only tangentially. STRONGLY CONNECTED is defined between two individuals when they overlap substantively. This can be succinctly defined in the full theory of mereotopology as in D 18. Recalling the notion of strong self-connectedness (SSC), a relation of strong connection can be defined between two m-individuals if their 
A mereotopological approach to the mass/count distinction

sum is strongly self-connected. Note that defining strongly connected in terms of simple overlap leaves something to be desired, as that would include cases of tangential overlap as being considered strongly connected. Here the interiors of the two individuals are constrained to be overlapping, a stronger condition. In contrast to Strongly Connected, one can define the relation that holds between individuals which are only tangentially connected, or EXTERNALLY CONNECTED (EXTC), where two m-individuals are connected, but their interiors do not overlap, as given in D 19. ${ }^{3}$

D 18 Strong $C(x, y)={ }_{d e f} \exists w \exists z(w \leq x \wedge z \leq y \wedge \operatorname{SSC}(w \oplus z))$

D $19 \operatorname{Ext} C(x, y)=C(x, y) \wedge \neg C(i x, i y)$

To these variations, I add, in a slight abuse of terminology, a notion of PROXIMATELY CONNECTED (PROXC), where two entities are not contiguous, but "sufficiently near". (To foreshadow, this is an appropriate connectedness relation to describe, for instance, swarming insects.) To establish this relation, a definition of distance must be given. I assume a standard distance function $d$, which given two entities, returns the distance between them. A relation which holds of entities which are 'proximate' to one another can be defined as in D 20. Of course, the aim is not to characterize nearness in any absolute sense, but relative to the entities under discussion. I assume that the relevant value of $n$ will be determined with respect to the predicate.

D $20 \operatorname{ProxC}(x, y)={ }_{\operatorname{def}} d(x, y) \leq n$

This concludes the addition of different varieties of connectedness that will be useful for characterizing different entity types with respect to their relation to different countability classes.

\subsection{Substances}

I now turn to the designation of substance terms such as water or iron. Part of the intuition behind the property of divisiveness, as well as Schwarzschild's (2011) or Chierchia's (1998b) analysis of non-countable nouns, is that pure substances such as oil, water or wood have the trait that when one refers to an m-individual, then simultaneously one refers to multiple instances. Yet, as reviewed in section 3.1, modeling this intuition using the property of divisiveness leads to the unwelcome consequence of the "minimal parts problem". The other route, collapsing substance

3 An equivalent to the external connection relation is used by Krifka (1998) to define "adjacency structures" which are used to model paths. 
terms with plural entities, captures this intuition but violates other intuitions, e.g., that substances are simply different from a collection of entities.

Within the framework developed here, the intuition that substances involve multiplicity in a certain sense can be satisfied, yet something still stronger can be said. Substances manifest the property that a given instance of, say, water, will overlap with other instances of water. In terms of the framework just developed, instances of a substance come strongly connected with other instances. In this sense, the presence of an instance of water implies the presence of another instance of water. Recalling that the converse of connected is separated, another way to fix the intuitions for this property is that no instance of a liquid/substance is separated from all the other instances of that liquid/substance. The relevant property is that each occurrence of water is locally strongly connected. ${ }^{4}$

The condition given in (7) states that substance predicates require their extension to be comprised of m-individuals which are strongly connected to other m-individuals of the same substance. This will be satisfied, for instance, by a section of a pool of water-it overlaps with other sections of the pool of water, which are again water. Also, since connectedness is implied by parthood, the whole pool of water is strongly connected to its parts which are water.

$$
\begin{aligned}
& \text { SUBSTANCE(P) } \rightarrow \forall x\left[P(x) \rightarrow \exists x^{\prime}\left[P\left(x^{\prime}\right) \wedge x^{\prime} \neq x \wedge \operatorname{StrongC}\left(x, x^{\prime}\right)\right]\right] \\
& \text { (If } P \text { is a substance predicate then all } m \text {-individuals that satisfy } P \text { are strongly } \\
& \text { connected to a distinct } m \text {-individual of the same substance.) }
\end{aligned}
$$

This condition will also be satisfied when taking the sum of two portions of water, say two pools - here supposing that an instance of water was the sum of two portions of water $a \oplus b$. Then our condition tells us that there are additional strongly connected m-indiviudals for each element of the sum-individual.

This condition on the semantics of substances has several advantages over the properties used in previous approaches. First, unlike cumulativity, the condition in (7) distinguishes substance predicates from nouns with plural denotations. Plural nouns such as boys do not satisfy the condition of having their referents being (locally) strongly connected. Second, the difficulties facing divisiveness are avoided under this account. The difficulty was that many substance nouns do not lend themselves to being infinitely divisible into the same type of stuff. Soup, for instance, may contain meatballs that, while part of the soup, are not in themselves soup, contrary to what divisiveness predicts. The characterization of substance terms given in (7), however, accords with this scenario: any individual portion of a soup which in itself

4 'Locally' connected indicates that not every instance of water is connected, since clearly water may appear parceled out into puddles, rivers, glasses and so on; but locally, an instance of water is always connected to another instance of water, where the second instance may contain or be contained in the first. 
qualifies as soup will be strongly connected to another such individual. Yet, there is no commitment to infinite part-taking, the problematic aspect of divisiveness.

A final remark concerns the observation that there is no (natural) individuating standard for substance entities (Cartwright 1975; Quine 1960). It turns out that in the system developed here establishing a "minimal instance" for a substance term leads to contradiction, which is a welcome result. I take a minimal instance to be one which (i) does not contain another entity of the same sort (which is the atomicity property) and (ii) is able to be isolated from other instances of the entity (which is the property of being separated from other instances, in other words not being connected to other instances). A predicate such as boy will clearly satisfy having a minimal instance. Yet, an entity falling under a substance predicate, assuming that it obeys the condition given in (7), cannot satisfy these conditions. Suppose there was a minimal instance of water, water $_{1}$. In order to satisfy (7), this instance

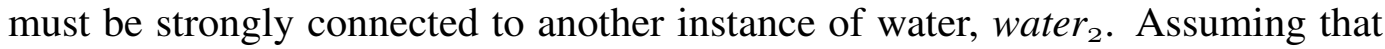
water $_{1}$ is strongly connected to water $_{2}$ by containing it violates the first condition of being a minimal instance, whereas assuming that they are connected in any other manner (viz. overlap) violates the second. Thus, assuming that a substance term has a minimal instance leads to a contradiction. In sum, treating substance terms as designating entities which must be strongly connected to like entities avoids some of the classic problems facing divisiveness, yet still represents the core intuitions that one would want the semantic theory to represent.

\subsection{Aggregates}

Aggregates, such as sand or rice, are a hybrid, possessing at once minimal parts while also designating groups which come together in some manner. The mereotopological framework provides sufficient means to represent aggregates as a category of entities that differ from individuals or sums. I will discuss the two most prominent types of aggregates, what I have termed granular and collective aggregates. The core idea that I will pursue is that while aggregate terms designate minimal parts, and accordingly will designate sums of these minimal parts, the different connection relations can be exploited to designate "connected clusters" of the entities in question, ultimately capturing that, for instance, sand occurs as individual grains, as sums thereof, as well as in clusters.

To treat aggregates, two auxiliary notions must be defined. First, I give the relation TRANSITIVELY CONNECTED (TransitiveC) relative to a property in D 21. This relation allows us to state whether two entities are connected through a series of like entities. For instance, two grains of sand on opposite sides of a pile of sand are not externally connected to one another, but they are transitively (externally) connected through a series of grains of sand within the pile. 
D 21 Transitive $C(x, y, P, C, Z)={ }_{\text {def }} \forall z \in Z\left[P(z) \wedge\left(x=z_{1} \wedge y=z_{n}\right) \wedge C z_{1} z_{2} \wedge C z_{2} z_{3} \ldots \wedge\right.$ $\left.C z_{n-1} z_{n}\right]$ where $Z=\left\{z_{1}, z_{2}, \ldots, z_{n}\right\}$ (Transitively Connected)

( $x$ and $y$ are transitively connected relative to a property $P$, a connection relation $C$, and $a$ set of entities $Z$, when all members of $Z$ satisfy $P$ and $x$ and $y$ are connected through the sequence of $z_{i}$ s in $Z$.)

With the definition of transitive connection in hand, the notion of a connected cluster can be given in D 22. This definition provides a way to refer to collections of $\mathrm{m}$-individuals that are related by a particular connection relation, such as a pile of sand or a swarm of locusts. At the same time, this does not enforce taking maximal clusters - a desirable property as, for instance, a pile of sand, which is a clustered individual, includes many other clustered individuals.

D 22 Cluster $(x, P, C)=_{\text {def }} \exists Z\left[x=\bigoplus Z \wedge \forall z, z^{\prime} \in Z \exists Y\left[\right.\right.$ Transitive $\left.\left.C\left(z, z^{\prime}, P, C, Y\right)\right]\right]$ (Clustered Individual)

( $x$ is a cluster relative to a property $P$ and a connection relation $C$ iff $x$ is a sum of entities falling under the same property which are all transitively connected relative to some set $Y$ under the same property and connection relation.)

A general condition on aggregates is given in (8) below, which states that the denotational space of aggregate nouns includes MSSC individuals, clusters and sums. In (8), I use the notation $\mathscr{C}$ as a variable over connectedness relations, e.g., External Connectedness and Proximately Connected, and use $\mathrm{CLUSTER}_{\mathscr{C}}$ to represent the set of clustered individuals under the relevant connection relation $C, \mathrm{SUM}$ to represent the set of sum individuals and MSSC for the set of MSSC individuals.

$$
\mathrm{AGG}(\mathrm{P}, \mathrm{C}) \rightarrow \forall x\left[P(x) \rightarrow x \in \mathrm{CLUSTER}_{\mathscr{C}} \cup \mathrm{SUM} \cup \mathrm{MSSC}\right]
$$

(If $P$ is an aggregate predicate relative to a connection relation then all $m$-individuals that satisfy $P$ have cluster, MSSC or sum reference.)

The type of connection relation has been underspecified in the definition, but instantiating it with different connection types results in the different aggregate types discussed. A clustered individual under the External Connectedness relation designates a cluster of individuals which are connected by touching, which is an appropriate designation for a canonical instance of sand or rice. A clustered individual under the Proximately Connected relation specifies a group of individuals all within a particular distance of one another, for instance, berries which are all within one inch of each other. This serves as a representation of collective aggregates such as insects or berries, which do not appear in groups where each individual touches another, but where they are all at some distance from one another.

This definition is very permissive, but is useful as a general definition of aggregates. Sand, for example, will be true of single grains (MSSC individuals) or 
A mereotopological approach to the mass/count distinction

sums thereof, as well as clusters of sand (e.g., piles), which are clusters of externally connected individuals, or a combination of these types.

This section has developed the formal machinery to treat different entities types. I now turn to using the various formal distinctions in accounting for the grammatical number systems of English and Welsh.

\section{Number morphology}

Against the backdrop of the richer framework developed, the actual account of the semantics of number morphology is, as one would hope, rather simple. Three different types of elements are in play. First, nouns which allow number distinctions may vary in different languages as to what is designated by the basic form of a noun, e.g., that which is not morphologically marked by a number morpheme. For instance, an unmarked countable noun in English refers to single entities, while an unmarked noun in Welsh may refer to clustered individuals. As for the number morphemes, they vary both in (i) the nature of the operation, viz. pluralization or unitization, and (ii) the presuppositions in effect about the domains over which they operate. I first discuss the more familiar case of English and then proceed to Welsh.

\subsection{English}

The core result that one would like from a compositional account of the number morphology of English is an adequate treatment of where number morphology, viz. pluralization via $-s$, is permitted to occur and where it is not. For one part of this problem, namely treating countable nouns, I am able to follow traditional accounts of plurality with only minor modifications. The additional distinctions made in section 3 come into play for accounting for where plurality is disallowed: both nouns such as water and sand violate the prerequisites of plurality, but in different fashions.

Countable nouns in English, for which I will consider dog to be the parade example, do not require any radically different account from those used in the past, and I am able to maintain the standard view, with the exception that the property used is of course no longer atomicity but being a MSSC individual. Thus, we can state with, for instance, Link (1983) that the denotation of the singular form of $d o g$ is the property satisfied by singular entities, as given in (9), although MSSC is now the relevant property determining what a singular entity is.

$$
\llbracket \operatorname{dog} \rrbracket:=\lambda x_{O}\left[\mathrm{R}\left(x_{O}, \operatorname{Dog}\right) \wedge \operatorname{MSSC}\left(x_{O}\right)\right]
$$

The plural morpheme $-s$ can be treated as an operator, which presupposes a set of, 
in our case, MSSC individuals and then returns the set of sums. ${ }^{5}$ The presupposition associated with the operation is expressed following a period after the lambda expression, as in " $\lambda P$.Presupposition $[---]$ ". I also make use of the ${ }^{*}$ operator from Link (1983), which given a set, returns the sums of things in that set. This analysis of $-s$ is given in (10), which parallels Link's analysis of the plural.

$$
\llbracket-\mathrm{-s} \rrbracket:=\lambda P \lambda x . P_{M S S C}\left[P^{*}(x)-\operatorname{MSSC}(x)\right]=\lambda P . P_{M S S C}\left[P^{*}(x) \wedge x \in \mathrm{SUM}\right]
$$

Given this treatment of the plural, its incompatibility with substance predicates such as water is immediate: the plural morpheme requires MSSC individuals, which are not provided by substance predicates. ${ }^{6}$ Similarly, granular aggregate nouns in English such as sand, rice or gravel, like substance terms, designate the entire semilattice, as in (11). Again, pluralization fails, since there is not a set of MSSC individuals from which sum individuals can be formed, rather the denotation of the noun includes much more.

$$
\llbracket \text { sand } \rrbracket:=\lambda x_{O}\left[R\left(x_{O}, \text { Sand }\right) \wedge x_{O} \in \mathrm{CLUSTER} \mathrm{C}_{E} \cup \mathrm{SUM} \cup \mathrm{MSSC}\right]
$$

I now turn to Welsh, which exploits the denotational space of these nouns in a different way.

\subsection{Welsh}

The countability categories of nouns in Welsh can be treated in an analogous manner to the analysis of English, despite the fact that the inventory differs. The class of singular/plural nouns in Welsh admits of the exact same treatment as given for English nouns with a singular/plural distinction. The denotation of an individual predicate such as cadiar 'chair' and the plural morpheme -oul-au parallel the entries for their English counterparts and are given in (12) and (13), respectively. Similarly, nothing special needs to be said about substance terms in Welsh-they behave in the same manner as substance terms in English do.

$$
\begin{aligned}
& \llbracket \text { cadair } \rrbracket:=\lambda x_{O}\left[\mathrm{R}\left(x_{O}, \text { Chair }\right) \wedge x_{O} \in \mathrm{MSSC}\right] \\
& \llbracket \text {-ou/-au } \rrbracket:=\lambda P \lambda x . P_{M S S C}\left[P^{*}(x)-\operatorname{MSSC}(x)\right]=\lambda P \lambda x \cdot P_{M S S C}\left[P^{*}(x) \wedge x \in\right. \\
& \text { SUM }]
\end{aligned}
$$

5 I am here assuming the classical "exclusive" analysis of the plural, where singular entities are not included. Nothing crucial hinges on this for the points being made here about English or Welsh.

6 There may be polysemy through which a second representation for, e.g., water, is available that presupposes a set of MSSC individuals, such as servings of water. But the availability of this meaning is here taken to be a fact about lexicalization or, in some cases, coercion of noun meaning. 
A mereotopological approach to the mass/count distinction

The collective/unit class is the interesting case, but it too can be treated in a manner similar to the analysis of English. First, in the same way that unmarked count nouns in English are restricted to being interpreted as singular, collective/unit nouns in Welsh are in their basic form restricted to clustered individuals. The lexical entry for cacwn 'hornet' is given in (14), stating that cacwn designates entities which satisfy the property hornet and are clustered individuals by virtue of being proximately connected. The denotational space of the lexical entry does not include arbitrary sums. This is consistent with the discussion of Welsh in section 2, where it was noted that these nouns had a strong sense of designating a collection rather than an arbitrary set of individuals. Additionally, if arbitrary sums were included, it would be expected that these nouns could be directly modified by cardinal numbers-but these nouns only take cardinal modification once the singulative has been applied.

$$
\llbracket \text { cacwn } \rrbracket:=\lambda x_{O}\left[\mathrm{R}\left(x_{O}, \text { Hornet }\right) \wedge x_{O} \in \mathrm{CLUSTER}_{P C}\right]
$$

The singulative marker can then be treated as an operator as in (15). In parallel to the restriction on the plural morpheme which presupposes MSSC individuals, the singulative morpheme presupposes a set of clustered individuals. Given such a set of clustered individuals, it returns the parts which are MSSC, i.e., the units. Since the singulative presupposes clustered individuals, its application to count nouns such as cadiar 'chair' is correctly ruled out. Similarly, the application of the singulative would also be ruled out for substance nouns-although substance nouns possess clustered individuals, they do not possess MSSC-individuals which would serve as the output of the operation.

$$
\llbracket \text {-en/-yn } \rrbracket:=\lambda Q \lambda x \cdot Q_{\text {cluster }}[x \leq Q \wedge x \in \mathrm{MSSC}]
$$

As noted in section 2, collective nouns in Welsh may also allow the application of the plural following the singulative, as was given in (4). The definitions of the singulative and plural here allow for this possibility: the application of the singulative provides the MSSC individuals which in turn feed the application of the plural.

\section{Conclusion}

Taking a broader range of number systems, of which Welsh is just one instance, into account indicates that the semantic distinctions available in the classical mereological treatment of countability are not sufficient for a full treatment of countability. Enriching mereology with topological connectedness relations not only provides the expressivity to treat Welsh's collective/unit class, but resolves long-standing problems for the classical account, namely the "minimal parts" problem and difficulties with predicates such as fence. Future work should explore how the analysis given here may be extended both in terms of other grammatical systems as well as in terms of other regions of the lexicon, e.g., for eventive and abstract nouns. 
Grimm

\section{References}

Cartwright, Helen. 1975. Amounts and measures of amount. Noûs 9. 143-164. doi: $10.2307 / 2214598$.

Casati, R. \& A. C. Varzi. 1999. Parts and Places: The Structures of Spatial Representation. Cambridge, MA: MIT Press.

Chierchia, Gennaro. 1998a. Plurality of mass nouns and the notion of "semantic parameter". In Susan Rothstein (ed.), Events and Grammar, 53-104. Dordrecht: Kluwer Academic Publishers.

Chierchia, Gennaro. 1998b. Reference to kinds across languages. Natural Language Semantics 6. 339-405.

Goodman, Nelson. 1951. The Structure of Appearance. Cambridge, MA: Harvard University Press.

Grimm, Scott. 2012. Number and Individuation: Stanford University dissertation.

Jones, Morris \& Alan R. Thomas. 1977. The Welsh Language: Studies in its Syntax and Semantics. Cardiff: University of Wales Press for the Schools Council.

King, Gareth. 2003. Modern Welsh: A Comprehensive Grammar. Routledge.

Krifka, Manfred. 1989. Nominal reference, temporal constitution and quantification in event semantics. In Renate Bartsch, Johan van Benthem \& Peter van Emde Boas (eds.), Semantics and Contextual Expressions, 75-115. Dordrecht: Foris Publications.

Krifka, Manfred. 1998. The origins of telicity. In Susan Rothstein (ed.), Events and Grammar, 197-235. Dordrect: Kluwer.

Landman, Fred. 1989. Groups I. Linguistics and Philosophy 12. 559-605. doi:10.1007/BF00627774.

Link, Godehard. 1983. The logical analysis of plurals and mass terms: A latticetheoretical approach. In Rainer Bauerle, Christoph Schwarze \& Arnim von Stechow (eds.), Meaning, Use, and Interpretation of Language, Berlin: de Gruyter.

Moltmann, Frederieke. 1997. Parts and Wholes in Semantics. New York: Oxford University Press.

Pianesi, Fabio. 2002. 'Parts and wholes in semantics' by Friederike Moltmann. Linguistics and Philosophy 25. 97-120. doi:10.1023/A:1014347214919.

Prasada, Sandeep, Krag Ferenz \& Todd Haskell. 2002. Conceiving of entities as objects and stuff. Cognition 83. 141-165. doi:10.1016/S0010-0277(01)00173-1.

Quine, W. V. O. 1960. Word and Object. Cambridge, MA: MIT Press.

Rothstein, Susan. 2010. Counting and the mass/count distinction. Journal of Semantics 27. 343-397. doi:10.1093/jos/ffq007.

Schwarzschild, Roger. 2011. Stubborn distributivity, multiparticipant nouns and the count/mass distinction. In Suzi Lima, Kevin Mullin \& Brian Smith (eds.), North 
A mereotopological approach to the mass/count distinction

East Linguistics Society (NELS) 39, vol. 2, 661-678. Amherst, MA: GLSA. Smith, Barry. 1996. Mereotopology: A theory of parts and boundaries. Data and Knowledge Engineering 20. 287-303. doi:10.1016/S0169-023X(96)00015-8.

Soja, Nancy N., Susan Carey \& Elizabeth S. Spelke. 1991. Ontological categories guide young children's inductions of word meaning: Object terms and substance terms. Cognition 38. 179-211. doi:10.1016/0010-0277(91)90051-5.

Stolz, Thomas. 2001. Singulative-collective: Natural morphology and stable cases in Welsh number inflexion of nouns. Sprachtypologie und Universalienforschung 54(1). 52-76.

Taylor, Barry. 1977. Tense and continuity. Linguistics and Philosophy 1. 199-220. Wiggins, David. 1980. Sameness and Substance. Oxford: Blackwell.

Zucchi, Sando \& Michael White. 2001. Twigs, sequences and the temporal constitution of predicates. Linguistics and Philosophy 24. 223-270. doi:10.1023/A:1005690022190.

\author{
Scott Grimm \\ Departament de Traducció i Ciències del Llenguatge \\ Universitat Pompeu Fabra \\ Carrer de Roc Boronat, 138 \\ 08018 Barcelona, Spain \\ scott.grimm@upf.edu
}

